IFUSP-P 1083

\title{
Signals for Vector Leptoquarks in Hadronic Collisions
}

\author{
J. E. Cieza Montalvo * and O. J. P. Éboli ${ }^{\dagger}$ \\ Instituto de Física, Universidade de São Paulo \\ C.P. 20516, 01452-990 São Paulo, Brazil
}

(October 10, 2018)

\begin{abstract}
We analyze systematically the signatures of vector leptoquarks in hadronic collisions. We examine their single and pair productions, as well as their effects on the production of lepton pairs. Our results indicate that a machine like the CERN Large Hadron Collider (LHC) will be able to unravel the existence of vector leptoquarks with masses up to the range of $2-3 \mathrm{TeV}$.
\end{abstract}

Submitted to Phys. Rev. D 


\section{INTRODUCTION}

Many extensions of the Standard Model predict the existence of leptoquarks, which are color triplet particles carrying simultaneously leptonic and baryonic number and can have spin 0 or 1 . For instance, some composite models postulate a preonic sub-structure where quarks and leptons have some common constituents [1, 2]. These models exhibit a very rich spectrum which includes excited states of the known particles, as well as new particles possessing unusual quantum numbers, such as leptoquarks and dileptons. Leptoquarks are also present in extensions of the standard model that treat quarks and leptons in the same basis and, consequently, allow the existence of new particles mediating quark-lepton transitions. This class of models includes grand unified theories [3], technicolor models [4], and superstring-inspired models [5].

The production and signals of scalar leptoquarks have already been analyzed in the literature for $e p$ [6.7], hadronic [8], $e^{+} e^{-}$[9], and $e \gamma$ [10] colliders. However, previous studies of vector leptoquarks $(V)$ considered only $e^{+} e^{-}$[11,12] and ep colliders [7]. In this work, we study the capability of hadron colliders to unravel the existence of vector leptoquarks. We analyze their pair production via gluon-gluon and quark-antiquark fusion $(g(q)+g(\bar{q}) \rightarrow$ $V+\bar{V})$, single production in association with a lepton $\left(g+q \rightarrow V+\ell, \ell=e^{ \pm}, \mu^{ \pm}, \nu\right)$, and their effects in the production of lepton pairs $\left(q+\bar{q} \rightarrow \ell^{+}+\ell^{-}\right)$. Our results show that a machine like the LHC can expand considerably the range of leptoquark masses and couplings that can be explored.

It is interesting to notice that pair production of vector leptoquarks is essentially model independent since it is related to the strong interactions. On the other hand, single production and indirect effects are to a great extent model dependent because they are due to unknown interactions. Moreover, these three signals for vector leptoquarks are complementary since they allow us not only to reveal their existence but also to determine their properties such as mass, spin, and couplings to quarks and leptons.

There have been several direct searches for leptoquarks in accelerators. Analyzing the 
decay of the $Z$ into a pair of on-shell leptoquarks, the LEP experiments established a lower bound $M_{l q} \gtrsim 44 \mathrm{GeV}$ for scalar leptoquarks [13,14], and a similar bound should hold for vector ones. This limit can be improved by looking for $Z$ decays into an on-shell and an off-shell leptoquarks, resulting in $M_{l q} \gtrsim 65-73 \mathrm{GeV}$ [14]. The search for scalar leptoquarks decaying into an electron-jet pair in $p \bar{p}$ colliders constrained their masses to be $M_{l q} \gtrsim 113$ $\mathrm{GeV}$ [15]. Furthermore, the experiments at HERA [16] placed limits on their masses and couplings, leading to $M_{l q} \gtrsim 92-184 \mathrm{GeV}$ depending on the leptoquark type and couplings.

Leptoquarks are also tightly constrained by low energy experiments [17, 18, such as the proton lifetime measurements and the study of rare decays associated to flavor changing neutral currents. In order to avoid the strong low energy bounds we assumed that the leptoquark interactions conserve lepton and baryon number in addition to electric charge and color, and that their couplings are chiral [17]. Furthermore, leptoquarks that couple to more then one fermion generation are constrained to be very heavy, therefore, we shall study vector leptoquarks that couple only to one family.

For the sake of definiteness, we shall consider the vector leptoquarks inspired by the Abbott-Farhi model [2], which is a confining version of the Standard Model and is described by a Lagrangian which has the same general structure of the Standard Model one. Nevertheless, the choice of parameters is such that no spontaneous symmetry breaking occurs and the $S U(2)_{L}$ gauge interaction is confining. In this model, the physical left-handed fermions are bound states of a preonic scalar and a preonic left-handed fermion, while the vector bosons are $\mathrm{P}$-wave bound states of the scalar preons. The vector leptoquarks predicted by this model are color triplets with electric charge $Q_{V}=-2 / 3$ and weak isospin 0 .

This model cannot be analyzed perturbatively since it is strongly interacting in the energy scale of interest. Therefore, we shall parametrize the interaction between vector leptoquarks $\left(V_{\mu}^{a b}\right)$ and physical left-handed fermions by an effective Lagrangian [6] given by

$$
\mathcal{L}_{\text {int }}=-F \frac{e}{2 \sqrt{2} \sin \theta_{W}}\left(V_{\mu}^{a b^{\dagger}} \bar{L}^{a} \gamma^{\mu} L^{b}+\text { h.c. }\right)
$$

where $L^{a}$ are physical left-handed doublets, $a(b)$ is a flavor index, $\theta_{W}$ is the weak mixing 
angle, and the parameter $F$ is a measure of the strength of this interaction compared to the $W q \bar{q}^{\prime}$ vertex. The above Lagrangian is a prototype of a wide class of models [7] presenting the interaction of color triplet, charged vectors with $B$ and $L$ numbers. According to (1), vector leptoquarks couple to both upper or lower components of the lepton and quark doublets with the same strength.

We can estimate the size of the coupling $F$ imposing that unitarity is respected at tree level [19]. For instance, the process $e^{+} e^{-} \rightarrow V \bar{V}$ violates unitarity at high energies for arbitrary values of the couplings. However, this can be avoided by choosing $F=\sqrt{\left|Q_{V}\right|}=$ $\sqrt{\frac{2}{3}}[11]$.

Since the vector leptoquarks are color triplets, it is natural to assume that they interact with gluons. To obtain their couplings to gluons we substituted $\partial_{\mu}$ by the covariant derivative $D_{\mu}=\partial_{\mu}+i g_{s} \lambda_{j k}^{a} G_{\mu}^{a} / 2$ in the vector leptoquark kinetic lagrangian and introduced an anomalous color magnetic moment $\kappa$. For $\kappa \neq 1$, unitarity is not respected at tree level by this model. Therefore, we must either impose $\kappa=1$ or introduce a new cutoff mass scale $\Lambda$. We assumed that $\kappa=1$, since this leads to conservative estimates for the cross sections.

The main decay modes of vector leptoquarks are into pairs $\ell q$ and $\nu q^{\prime}$, thus, their signal is a charged lepton plus a jet, or a jet plus missing energy. Using the couplings given above we obtain that the width of a vector leptoquark is

$$
\Gamma_{V}=\frac{\alpha F^{2}}{4 \sin ^{2} \theta_{W}} M_{V}
$$

where we neglected all fermion masses and summed over all possible decay channels.

The outline of this paper is the following. Section II contains the study of the production of vector leptoquark pairs via quark-antiquark and gluon-gluon fusion, which leads to conservative estimates of the discover potential of vector leptoquarks in the next generation of hadronic colliders. In Sec. III we analyze the single production of vector leptoquarks through quark-gluon fusion, exhibiting our results for first and second generation vector leptoquarks. The analysis of the indirect signals for leptoquarks is contained in Sec. IV: One way to look for vector leptoquarks is through their effects on the production of lepton 
pairs at high energies, since they can be exchanged in the $t$-channel. Our conclusions are drawn in Sec. V.

\section{PAIR PRODUCTION OF VECTOR LEPTOQUARKS}

Pair production of new particles is, to a good approximation, a model independent process since it usually proceeds through well known strong and electroweak interactions. Consequently, this process leads to more reliable estimates of the possibility of discovering new physics. In a hadronic collider there are two main mechanisms for the production of vector leptoquark pairs: gluon-gluon fusion $(g+g \rightarrow V+\bar{V})$ and quark-antiquark fusion $(q+\bar{q} \rightarrow V+\bar{V})$.

In lowest order, the Feynman diagrams contributing to the production of vector leptoquark pairs via gluon-gluon fusion are the exchange of gluons in the s-channel, vector leptoquarks in the $t$ - and $u$-channels, and a contact interaction $(g g V V)$, being its cross section for $\kappa=1$ given by 20]

$$
\begin{aligned}
\frac{d \hat{\sigma}_{g g}}{d \cos \theta}= & \frac{\pi \alpha_{s}^{2}}{16} \frac{\beta}{\hat{s}}\left(\frac{4}{3}-3 \frac{t_{M} u_{M}}{\hat{s}^{2}}\right) \times \\
& {\left[3+2 \frac{\hat{s}^{2}}{t_{M} u_{M}}\left(\frac{\hat{s}^{2}}{t_{M} u_{M}}-2\right)+6 \frac{\hat{s} M_{V}^{2}}{t_{M} u_{M}}\left(\frac{\hat{s} M_{V}^{2}}{t_{M} u_{M}}-1\right)\right], }
\end{aligned}
$$

where $\sqrt{\hat{s}}$ is the invariant mass of the subprocess, $t_{M}=-\hat{s}(1-\beta \cos \theta) / 2, u_{M}=$ $-\hat{s}(1+\beta \cos \theta) / 2, \theta$ is the angle between the vector leptoquark and the incident gluon

in the subprocess center of mass frame, and $\beta=\sqrt{1-4 M_{V}^{2} / \hat{s}}$ is the leptoquark velocity in this frame. This cross section has a good high energy behavior and, for large values of $\hat{s}$, it approaches a constant value proportional to $1 / M_{V}^{2}$ due to the exchange of a vector particle in the $t$-channel.

The quark-antiquark fusion production of vector leptoquark pairs takes place by the exchange of a gluon in the $s$-channel and a lepton in the $t$-channel. However, this last contribution is expected to be much smaller than the gluon exchange since the leptoquark couplings to quarks and leptons are expected to have a weak interaction strength - that 
is, it is natural to have $F$ of the order of the unit. Therefore, we restrict ourselves to the $s$-channel production only [21], and the elementary cross section for this process is [20]

$$
\begin{aligned}
& \frac{d \hat{\sigma}_{q \bar{q}}}{d \cos \theta}=\frac{9 \pi \alpha_{s}^{2}}{2} \frac{\beta}{\hat{s}} \times \\
& \quad\left\{\frac{1}{4}\left(1+\beta^{2}\right)^{2} \frac{\hat{s}}{M_{V}^{2}}-1+\beta^{2}+\frac{1}{4}\left(\frac{t_{M} u_{M}}{\hat{s}^{2}}-\frac{M_{V}^{2}}{\hat{s}}\right)\left[8+\frac{1}{4}\left(1+\beta^{2}\right)^{2} \frac{\hat{s}^{2}}{M_{V}^{4}}\right]\right\} .
\end{aligned}
$$

In order to obtain the inclusive total cross section for the production of pairs of vector leptoquarks $(p p \rightarrow V \bar{V} X)$ we must fold the proton structure functions with the elementary cross sections above

$$
\begin{aligned}
\sigma_{\text {pair }}=\int d x_{1} d x_{2}[ & g\left(x_{1}, Q^{2}\right) g\left(x_{2}, Q^{2}\right) \hat{\sigma}_{g g}(\hat{s}) \\
& \left.+\left(q\left(x_{1}, Q^{2}\right) \bar{q}\left(x_{2}, Q^{2}\right)+q\left(x_{2}, Q^{2}\right) \bar{q}\left(x_{1}, Q^{2}\right)\right) \hat{\sigma}_{q \bar{q}}(\hat{s})\right],
\end{aligned}
$$

where $g$ and $q$ are the relevant gluon and quark structure functions, and $\hat{s}=x_{1} x_{2} s$ with $\sqrt{s}$ being the machine center of mass energy. Figure 1 exhibits our results for the total cross section for the production of vector leptoquark pairs as a function of its mass at the LHC (taking $\sqrt{s}=15.4 \mathrm{TeV}$ ), where we imposed a rapidity cut $\left|y_{V(\bar{V})}\right|<2.5$ and used the set I of structure functions given in Ref. [22] evaluated at the scale $Q^{2}=\hat{s}$. As expected, the gluon-gluon-fusion contribution dominates over the quark-antiquark-fusion one at such high energies due to the large gluon-gluon luminosity. Notice that the above results hold true for any kind of vector leptoquark since we used only their strong interaction properties in the calculations.

The signal for leptoquark pair production is either $j j \ell^{+} \ell^{-}(j=$ jet $), j j \ell^{ \pm} p_{T}$, or $j j p_{T}$, where $\ell^{ \pm}=e^{ \pm}$or $\mu^{ \pm}$. Certainly the last signal will be emersed in a QCD background and will be very hard to extract. The other two, mainly the first, have a very good chance to be observed since they have the striking feature of producing charged lepton-jet pairs with a given invariant mass, however, a careful studied of the QCD and electroweak backgrounds is needed to know whether this kind of events can be seen or not [23]. Nevertheless, in order to have a rough estimate of the potential of the LHC to unravel the existence of vector 
leptoquarks via their pair production, we required the production of 100 of such pairs per year. Taking into account the expected integrated luminosity for the LHC $\left(10^{5} \mathrm{pb}^{-1} / \mathrm{yr}\right)$, we obtain that this machine will be able to discover first and second generation vector leptoquarks with masses up to $2.1 \mathrm{TeV}$.

\section{SINGLE PRODUCTION OF VECTOR LEPTOQUARKS}

The production of a single leptoquark in a hadronic collider must be associated with a lepton due to lepton number conservation. The subprocess $q+g \rightarrow V+\ell$ proceeds through the exchange of a leptoquark in the $t$-channel and a quark in the $s$-channel. This is a model dependent reaction since it involves unknown leptoquark-quark-lepton interactions. Using the model described in the introduction, it is easy to obtain that the elementary cross section for this process is

$$
\begin{aligned}
& \frac{d \hat{\sigma}_{\text {single }}}{d \hat{t}}=\frac{\pi \alpha \alpha_{s} F^{2}}{12 \sin ^{2} \theta_{W} M_{V}^{2}} \frac{1}{\hat{s}^{2}(\hat{s}+\hat{t})^{2}} \times \\
& \left\{2 \beta^{2} \hat{s}^{3}(3-2 \beta)+\hat{s}^{2} \hat{t}\left(-6+15 \beta-2 \beta^{2}-2 \beta^{3}\right)+\hat{s} \hat{t}^{2}\left(-1+8 \beta-2 \beta^{2}\right)+\hat{t}^{3}(1+\beta)\right\},
\end{aligned}
$$

where $\hat{t}=M_{V}^{2}-\left(\hat{s}+M_{V}^{2}\right)(1-\beta \cos \theta) / 2, \theta$ is the angle between the vector leptoquark and the incident quark in the subprocess center of mass frame, and $\beta=1-M_{V}^{2} / \hat{s}$ is the leptoquark velocity in this frame.

In order to obtain the total cross section for the inclusive process $p p \rightarrow V \ell X$ we must evaluate

$$
\sigma_{\text {single }}=\int d x_{1} d x_{2}\left[g\left(x_{1} ; Q^{2}\right) q\left(x_{2}, Q^{2}\right)+g\left(x_{2} ; Q^{2}\right) q\left(x_{1} ; Q^{2}\right)\right] \hat{\sigma}_{\text {single }}\left(x_{1} x_{2} s\right)
$$

where $g$ is the gluon structure function and $q$ is the density of down (up) quarks for $\ell=e^{ \pm}$ $\left(\ell=\nu_{e}\right)$ assuming a first generation leptoquark. In the case of a second generation leptoquark, $q$ is the strange (charm) structure function for $\ell=\mu^{ \pm}\left(\ell=\nu_{\mu}\right)$. Figure 2 summarize our results for the total cross section for the production of a vector leptoquark in association with charged leptons and neutrinos, where we imposed a rapidity cut on the charged 
particles $\left|y_{V\left(\ell^{ \pm}\right)}\right|<2.5$ and considered first and second generation vector leptoquarks. As expected, the cross section for producing pairs $V \nu_{e}$ is approximately a factor of 2 larger than the one for the production of $V e^{ \pm}$since they are associated to the up and down structure functions respectively. Since the strange content of the proton is larger than the charm one, the cross section for production of pairs $V \mu^{ \pm}$is larger than the one for pairs $V \nu_{\mu}$.

Taking into account that the decay of the leptoquark can give rise to a pair electron-jet or a jet plus missing energy, three different signals are possible for its single production: $j \ell^{+} \ell^{-}, j \ell^{ \pm} \not p_{T}$, and $j \not p_{T}$, where $\ell^{ \pm}=e^{ \pm}$or $\mu^{ \pm}$. This last signal has an incisive feature since it is a monojet event. However, it is going to be buried in the background produced by standard-model processes, like the production of $Z(\rightarrow \nu \bar{\nu})$ associated with a jet, which are expected to have a much higher cross sections [24]. On the other hand, the other two possible signals are very striking since they exhibit pairs $\ell^{ \pm} j$ with (approximately) the same invariant mass. A priori, it is hard to estimate the background for these signals since it is due to the QCD production of heavy quarks, or $W$ and $Z$ in association with jets, thus requiring further Monte Carlo work to determine their size [23].

In order to have an approximate idea of the potential of the LHC to discover vector leptoquarks via their single production, we required 100 events per year exhibiting a leptoquark decaying into a $\ell^{ \pm} j$ pair. Using the expected luminosity for the LHC and that the branching ratio of vector leptoquarks to $\ell^{ \pm} j$ pairs is $1 / 2$, we obtain that the LHC will able to probe first (second) generation vector leptoquark masses up to 2.7, 3.0, and 3.5 (1.9, 2.1, and 2.4) for couplings $F=0.5, \sqrt{2 / 3}$, and 1.5 respectively.

\section{INDIRECT SIGNALS FOR VECTOR LEPTOQUARKS}

The existence of leptoquarks can also be investigated through their effects as an intermediate state in the inclusive dilepton production $\left(p p \rightarrow \ell^{+} \ell^{-} X\right)$, where they lead to a new $t$-channel contribution in addition to the usual exchange of $\gamma$ and $Z$ in the $s$-channel. The

color averaged cross section for the subprocess $q \bar{q} \rightarrow \ell^{+} \ell^{-}$is, in the center of mass of the 
subprocess,

$$
\begin{aligned}
\frac{d \hat{\sigma}}{d \Omega}(\hat{s})= & \frac{\alpha^{2}}{12 \hat{s}}\left\{Q^{2}\left(1+\cos ^{2} \theta\right)+\frac{1}{16 \sin ^{4} \theta_{W} \cos ^{4} \theta_{W}} \frac{\hat{s}^{2}}{\left(\hat{s}-M_{Z}^{2}\right)^{2}+\Gamma_{Z}^{2} M_{Z}^{2}}\right. \\
& \times\left[\left(C_{V}^{e}{ }^{2}+C_{A}^{e 2}\right)\left(C_{V}^{q 2}+C_{A}^{q 2}\right)\left(1+\cos ^{2} \theta\right)+8 C_{V}^{e} C_{A}^{e} C_{V}^{q} C_{A}^{q} \cos \theta\right] \\
& -\frac{Q}{2 \sin ^{2} \theta_{W} \cos ^{2} \theta_{W}} \frac{\hat{s}\left(\hat{s}-M_{Z}^{2}\right)}{\left(\hat{s}-M_{Z}^{2}\right)^{2}+\Gamma_{Z}^{2} M_{Z}^{2}}\left[C_{V}^{e} C_{V}^{q}\left(1+\cos ^{2} \theta\right)+2 C_{A}^{e} C_{A}^{q} \cos \theta\right] \\
& +\frac{F^{2}}{\sin ^{2} \theta_{W}} \frac{(1+\cos \theta)^{2}}{\cos \theta-\eta}\left[\frac{F^{2}}{4 \sin ^{2} \theta_{W}} \frac{1}{\cos \theta-\eta}+\frac{Q}{2}\right. \\
& \left.\left.-\frac{1}{8 \sin ^{2} \theta_{W} \cos ^{2} \theta_{W}}\left(C_{V}^{q}+C_{A}^{q}\right)\left(C_{V}^{e}+C_{A}^{e}\right) \frac{\hat{s}\left(\hat{s}-M_{Z}^{2}\right)}{\left(\hat{s}-M_{Z}^{2}\right)^{2}+\Gamma_{Z}^{2} M_{Z}^{2}}\right]\right\}
\end{aligned}
$$

where $M_{Z}$ is the mass of the $Z$ boson and $\eta=1+2 M_{V}^{2} / s$. According to our conventions the charge of a quark is $Q e(e>0), C_{V}=I_{z}-2 Q \sin ^{2} \theta_{W}$, and $C_{A}=I_{z}$.

The exchange of a vector particle in the $t$-channel modifies the high energy behaviour of this subprocess: within the scope of the standard model this elementary cross section decreases as the center of mass energy increases, however, the new contribution alters this behaviour, yielding a constant cross section at high energies

$$
\sigma_{\text {limit }}\left(q \bar{q} \rightarrow \ell^{+} \ell^{-}\right) \simeq \frac{\pi \alpha \alpha_{s} F^{4}}{12 \sin ^{4} \theta_{W} M_{V}^{2}}
$$

The effect of this modification to the process $p p \rightarrow e^{+} e^{-} X$ due to a first generation vector leptoquark can be seen on Fig. 3, which displays the invariant mass distribution of dilepton pairs at the LHC for a vector leptoquark of mass $M_{V}=1 \mathrm{TeV}$ and several values of the coupling F. From this figure we can see that the presence of the leptoquark leads to a dramatic increase of the production of dilepton pairs at high invariant masses.

In order to estimate the capability of the LHC to search for vector leptoquarks, we evaluated the total cross section for the production of dileptons with an invariant mass greater than $300 \mathrm{GeV}$, exhibiting our results for first and second generation vector leptoquarks in Figs. 1 and 5 respectively. Demanding that the anomalous production of high invariant mass dileptons have a statistical significance of $5 \sigma$, we obtain that the largest first (second) generation vector leptoquark mass accessible at the LHC is $1.3,2.1$, and 4.0 (0.8, 1.4, and 2.6) TeV assuming that $F=0.5, \sqrt{2 / 3}$, and 1.5 respectively. 


\section{CONCLUSIONS}

The discover of leptoquarks is without any doubt a striking signal for the existence of life beyond the standard model. In this work we analyzed the potential of hadronic colliders, like the LHC, to unravel the existence of vector leptoquarks. Our results for the discovery

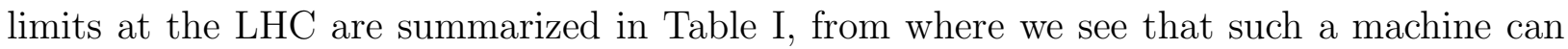
extend considerably the range of leptoquark masses and couplings that can be probed.

It is interesting to notice that the three signals for vector leptoquarks that we studied are complementary, i.e. their analysis allow us to shed some light on the leptoquark type and its couplings to quarks and leptons. For instance, the pair production, since is basically model independent, can be used to establish their existence. On the other hand, the single production rates, together with the knowledge of the charged lepton observed from their decays, can be used to determine to which quarks the leptoquarks couple. Moreover, the

production of high invariant mass lepton pairs can further confirm the signal and help to pinpoint the value of the couplings between leptoquarks, quarks and leptons.

Note added: During the time that we were writing this work we became aware of Ref. [26] that analyzes the vector leptoquark pair production in hadronic colliders. We verified that our results for this process agree.

\section{ACKNOWLEDGMENTS}

One of us (O.J.P.E.) would like to thank the kind hospitality of the Institute for Elementary Particle Research, University of Wisconsin-Madison, where the final part of this work was done. We are also grateful to M. C. González-García for a critical reading of the manuscript. This work was partially supported by Conselho Nacional de Desenvolvimento Científico e Tecnológico $(\mathrm{CNPq})$, and Fundação de Amparo à Pesquisa do Estado de São Paulo (FAPESP). 


\section{REFERENCES}

* E-mail address: CIEZA@USPIF.IF.USP.BR (internet) or 47602::CIEZA (decnet).

$\dagger \quad$ E-mail address: EBOLI@USPIF.IF.USP.BR (internet) or 47602::EBOLI (decnet).

[1] For a review see, W. Buchmüller, Acta Phys. Austr. Suppl. XXVII, 517 (1985).

[2] L. Abbott and E. Farhi, Phys. Lett. 101B, 69 (1981); Nucl. Phys. B189, 547 (1981).

[3] See, for instance, P. Langacker, Phys. Rep. 72, 185 (1981).

[4] S. Dimopoulos, Nucl. Phys. B168, 69 (1981); E. Farhi and L. Susskind, Phys. Rev. D20, 3404 (1979); J. Ellis et. al., Nucl. Phys. B182, 529 (1981).

[5] J. L. Hewett and T. G. Rizzo, Phys. Rep. 183, 193 (1989).

[6] J. Wudka, Phys. Lett. 167B, 337 (1986); M. A. Doncheski and J. L. Hewett, Z. Phys. C56, 209 (1992).

[7] W. Buchmüller, R. Rückl, and D. Wyler, Phys. Lett. 191B, 442 (1987).

[8] O. J. P. Éboli and A. V. Olinto, Phys. Rev. D38, 3461 (1988); J. L. Hewett and S. Pakvasa, Phys. Rev. D37, 3165 (1988).

[9] J. L. Hewett and T. G. Rizzo, Phys. Rev. D36, 3367 (1987); J. L. Hewett and S. Pakvasa, Phys. Lett. 227B, 178 (1987).

[10] O. J. P. Éboli et al., Phys. Lett. 311B, 147 (1993); H. Nadeau and D. London, Phys. Rev. D47, 3742 (1993).

[11] J. E. Cieza Montalvo and O. J. P. Éboli, Phys. Rev. D47, 837 (1993).

[12] J. Blümlein and R. Rückl, Phys. Lett. 304B, 337 (1993).

[13] L3 Collaboration, B. Adeva et al., Phys. Lett. 261B, 169 (1992); OPAL Collaboration, G. Alexander et al., Phys. Lett. 263B, 123 (1992); DELPHI Collaboration, D. Decamp et al., Phys. Rep. 216, 253 (1993). 
[14] DELPHI Collaboration, P. Abreu et al., Phys. Lett. 316B, 620 (1993).

[15] CDF Collaboration, F. Abe et al., Fermilab-Pub-93-070-E.

[16] ZEUS Collaboration, M. Derrick et al., Phys. Lett. B306, 173 (1993); H1 Collaboration, I. Abt et al., Nucl. Phys. B396, 3 (1993).

[17] W. Buchmüller and D. Wyler, Phys. Lett. 177B, 377 (1986); O. Shenkar, Nucl. Phys. B204, 375 (1982); B206, 276 (1982).

[18] S. Davidson, D. Bailey, and B. A. Campbell, preprint CfPA 93-TH-29.

[19] J. M. Cornwall, D. N. Levin, and G. Tiktopoulos, Phys. Rev. D10, 1145 (1974).

[20] G. V. Borisov, Yu. F. Pirogov, and K. R. Rudakov, Z. Phys. C36, 217 (1987).

[21] Taking into account only the $s$-channel gluon exchange spoils the high energy behaviour of this cross section. The cure to this problem requires the introduction of further interactions since all kind of quarks participate in this reaction. We shall neglect this fact, assuming that these new contributions are important only at very high energy where the structure function of the proton is negligible.

[22] D. Duke and J. Owens, Phys. Rev. D30, 49 (1984). We verified that the use of other parametrizations for the proton structure functions does not modify our results significantly.

[23] T. S. L. Lungov, O. J. P. Éboli, and R. Zukanovich Funchal, in preparation.

[24] See for instance, E. Eichten et al., Rev. Mod. Phys. 56, 579 (1984).

[25] In this estimate we considered only the irreducible background coming from the exchange of a $\gamma$ or a $Z$ in the $s$-channel. However, there are further backgrounds such as the production of heavy quark pair which should be included in a more accurate analysis. 
[26] J. L. Hewett et al., preprint ANL-HEP-CP-93-52. 


\section{TABLES}

\begin{tabular}{||r|c|c|c||}
\hline \hline$F$ & $V \bar{V}$ & $V \ell$ & $\ell^{+} \ell^{-}$ \\
\hline 0.5 & $2.1 / 2.1$ & $2.7 / 1.9$ & $1.3 / 0.8$ \\
$\sqrt{\frac{2}{3}}$ & $2.1 / 2.1$ & $3.0 / 2.1$ & $2.1 / 1.4$ \\
1.5 & $2.1 / 2.1$ & $3.5 / 2.4$ & $4.0 / 2.6$ \\
\hline \hline
\end{tabular}

TABLE I. Maximum first/second generation vector leptoquark masses that can be probed at the LHC for pair $(V \bar{V})$, single $(V \ell)$, and dilepton $\left(\ell^{+} \ell^{-}\right)$production, assuming an integrated luminosity of $10^{5} \mathrm{pb}^{-1} / \mathrm{yr}$. 


\section{FIGURES}

FIG. 1. Cross section for the production of vector leptoquarks pairs as a function of $M_{V}$ at the LHC: the dashed, dotted, and solid lines stand for $q \bar{q}, g g$ and total contributions. We imposed the rapidity cut $\left|y_{V}\right|<2.5$.

FIG. 2. Total cross section for the associated production of vector leptoquarks and leptons $\left(e^{ \pm}\right.$,

$\mu^{ \pm}, \nu_{e}$, and $\left.\nu_{\mu}\right)$ as a function of $M_{V}$ at the LHC. The dashed and solid (dotted and dash-dotted) stand for the production of a first (second) generation leptoquark in association with $e^{ \pm}$and $\nu_{e}$ $\left(\mu^{ \pm}\right.$and $\left.\nu_{\mu}\right)$ respectively. We imposed the rapidity cut $\left|y_{V \ell^{ \pm}}\right|<2.5$.

FIG. 3. Invariant mass distribution for $e^{+} e^{-}$pairs at the LHC: the solid, dotted, dashed, and dot-dashed lines stand for $F=0$ (standard model), $0.5, \sqrt{2 / 3}$, and 1.5 respectively. We assumed a first generation leptoquark of mass $M_{V}=1 \mathrm{TeV}$ and imposed that $\left|y_{e^{ \pm}}\right|<2.5$.

FIG. 4. Total cross section for the production of $e^{+} e^{-}$pairs as a function of $M_{V}$ at the LHC, satisfying $M_{e^{+} e^{-}}>300 \mathrm{GeV}$ and $\left|y_{e^{ \pm}}\right|<2.5$. Conventions are the same as in Fig. 3

FIG. 5. Same as in Fig. 团 but for $\mu^{+} \mu^{-}$pairs. 
This figure "fig1-1.png" is available in "png" format from: http://arxiv.org/ps/hep-ph/9311297v1 
This figure "fig1-2.png" is available in "png" format from: http://arxiv.org/ps/hep-ph/9311297v1 
This figure "fig1-3.png" is available in "png" format from: http://arxiv.org/ps/hep-ph/9311297v1 
This figure "fig1-4.png" is available in "png" format from: http://arxiv.org/ps/hep-ph/9311297v1 
This figure "fig1-5.png" is available in "png" format from: http://arxiv.org/ps/hep-ph/9311297v1 